\title{
Construir para deconstruir y volver a construir: elaboración colaborativa de un diccionario escolar de ciencias ${ }^{1}$
}

\section{Build to deconstruct and re-construct: collaborative development of a school science dictionary}

\author{
Rosa Estopà \\ Institut Universitari de Lingüistica Aplicada, Universitat Pompeu Fabra \\ rosa.estopa@upf.edu
}

RESUMEN • Este artículo presenta la metodología colaborativa utilizada en la construcción de un diccionario escolar de ciencias para niños de 5 a 8 ańos, basada en un corpus de definiciones de nińos. Durante 3 años se trabajó con 500 niños que definieron y dibujaron los términos más básicos de la ciencia. A partir del corpus, seleccionamos solo la información acumulativa científicamente positiva y así elaboramos las definiciones sin añadir nueva información. Finalmente, el artículo muestra la adecuación del diccionario a través de los resultados de una prueba de comprensión realizada con 160 escolares. Estos resultados permiten continuar con proyectos que trabajen el lenguaje junto al conocimiento científico desde los inicios: no hay ciencia sin palabras para referirse a ella ni sin palabras para comunicarla.

PALABRAS CLAVE: diccionarios escolares; corpus escolar; terminología escolar; ciencia, lengua.

\begin{abstract}
This paper presents the collaborative methodology used in the creation of a school dictionary of science for children aged between 5 and 8 years, based on a corpus of definitions given by children in the Catalan language. The work was conducted over a period of 3 years with 500 children who defined and described the most basic terms of science. From the corpus, we selected only the accumulated information which was scientifically positive and thus produced definitions without adding new information. Finally, the article demonstrates the suitability of the dictionary through the results of a lexical comprehension test carried out with 160 students. These results allow the carrying out of future plans which work the language with scientific knowledge from the beginnings: there is not science without words to represent it or to communicate it.
\end{abstract}

KEYWORDS: dictionaries for children; children's corpus; academic terminology; science; language.

Fecha de recepción: octubre 2013 • Aceptado: abril 2014

1. Este artículo se ha redactado en el marco del proyecto «Jugando a definir la ciencia», financiado por la Fundación Espańola para la Ciencia y la Tecnología, Programa de Cultura Científica y de la Innovación, 2011, FECYT-FCT-11-2501; IP: Rosa Estopà: «Jugando a definir la ciencia (2): Laboratorios de palabras» (Fundación Española para la Ciencia y la Tecnología, Programa de Cultura Científica y de la Innovación 2012, FECYT-FCT-12-4482; IP: Rosa Estopà), y por el Departament d'Economia i Coneixement, Secretaria d'Universitats i Recerca, 2012, 2012ACDS00050, IP: Rosa Estopà: «Jugant amb les paraules de la ciència». Para más información se puede consultar la página web del proyecto: http://defciencia.iula.upf.edu. Agradezco sinceramente la lectura del artículo a Segundo Moyano e Iria da Cunha.

Estopà Bagot, R. (2014) Construir para deconstruir y volver a construir: elaboración colaborativa de un diccionario escolar de Ciencias. Enseñanza de las Ciencias, 32.3, 


\section{INTRODUCCIÓN}

La ciencia se representa, se transfiere, se explica y se aprende a través de palabras especializadas, también conocidas como términos. El acceso al conocimiento especializado permite el uso adecuado y preciso de la terminología (Estopà y Valero, 2002). El proceso de expertización en una especialidad, sin embargo, es muy progresivo y se inicia en los primeros ańos de vida de una persona (Vigotski, 1962; Carretero, 1993; Golombek, 2006). Así, a medida que el conocimiento sobre un concepto va aumentando, el significado del término que lo designa va también evolucionando. Izquiero y Aliberas (2004) ramarcan que "pretender fijar de una vez por todas un significado es suprimirle su dinamismo, su capacidad de transformación y de adaptación y convertirlo en un fósil cognitivo que ya no sirve para pensar». Por eso, trabajar el lenguaje conjuntamente con el conocimiento científico desde los inicios es crucial para cualquier futura profesión científica, ya que no hay ciencia sin palabras para referirse a ella, pero tampoco sin palabras para comunicarla (Gutiérrez, 1998).

De hecho, en las clases en las que se imparte ciencia, tanto profesores como alumnos suelen pasarse mucho tiempo escuchando, leyendo y escribiendo acerca de ella (Lemke, 1997; Watt, 1989). Los mensajes orales y escritos reflejan maneras científicas de aproximarse a los objetos, hechos, acciones y teorías científicas. Izquierdo y Aliberas (2004: 117) alertan de la existencia de graves deficiencias comunicativas cuando los nińos hablan de ciencia. Y todavía es frecuente la simple respuesta de que «los problemas de lengua los debe resolver el profesorado de lengua», afirmación de la que discrepamos, ya que el trabajo holístico en la escuela potencia la interdependencia natural que existe entre palabras (lengua) y conocimiento (ciencia).

A menudo los niños y las niñas, también los muy pequeños, preguntan (y se preguntan) sobre el significado de palabras especializadas: ¿qué es un cráter?, ¿para qué sirve un microscopio?, ¿de qué color es nuestro cerebro?, ¿es lo mismo el universo que el espacio?, ¿qué significa neurona?... Trabajar el vocabulario especializado es una tarea prioritaria desde las primeras etapas de la enseńanza (Mallart, 2002; Ilari, 2008). Todo profesor que se proponga ayudar al alumno a ser más competente en el conocimiento y en la práctica de una lengua valora la importancia de usar el diccionario en el aula -aunque luego le pueda surgir la duda de qué obra de referencia utilizar e incluso de si para ello hay que usar el diccionario tradicional-. Sin embargo no siempre este axioma se puede traspasar a la enseñanza del conocimiento científico. Y, en cambio, en la explicación de las matemáticas, el medio, la historia, etc., el diccionario -y el trabajo con el vocabulario- puede tener la misma relevancia que en la clase de lengua:

Given the importance background knowledge and the fact that vocabulary is such an essential aspect of it, one of the most crucial services that teachers can provide, particularly for students who do not come from academically advantage backgrounds, is systematic instruction in important academic terms (Marzano y Pickering, 2005: 3).

Según estos autores, "without a basic knowledge of these terms, students will have difficulty understanding information they read or hear. Knowledge of important terms is critical to understanding any subject» (Marzano y Pickering, 2005: 2-3). Otros autores, como Locke (1992), Lemke (1997) e Izquierdo y Aliberas (2004), también defienden que debe enseñarse a solucionar de manera explícita las dificultades lingüísticas que se producen cuando se habla de ciencia en clase.

En este sentido, Carsleton y Marzano (2010: 1) establecieron una escala de seis etapas para trabajar el vocabulario académico en el aula, a las que nos referimos con el término escala Tennesee (Estopà, 2013). El primer nivel de la escala Tennesee consiste en proporcionar una descripción, explicación o ejemplo de cada nuevo término. Para este primer peldaño, el diccionario -que es el libro que explica qué significan las palabras de una lengua o de un determinado tema- debería ser uno de los recursos básicos de consulta en el aula. 
No obstante, muchas lenguas - como es el caso del castellano, del catalán, del gallego o del vascono disponen de diccionarios para todos los niveles escolares. En el marco de la lexicografía escolar en catalán -y también en las demás lenguas mencionadas- existe un vacío de diccionarios para los niños y niñas que se están iniciando en la alfabetización (o de los llamados, por especialistas como Prado (2000), diccionarios infantiles). De hecho, en las escuelas del Estado español el diccionario no se introduce curricularmente hasta tercero, y sobre todo se trabaja en cuarto y quinto de primaria. Sin embargo, esta situación no se da en todos los sistemas escolares. ${ }^{2}$ Por ejemplo, destacamos la política pedagógica actual de Brasil.

En 2006, el Gobierno de Brasil, en el Programa Nacional do Livro Didáctico (PNLD), incluyó de manera prioritaria una comisión para la evaluación y selección de diccionarios escolares. ${ }^{3}$ Esta comisión distinguió tres tipos de diccionarios generales escolares a partir del criterio del público destinatario (Carvalho y Bagno, 2011) (Kriguer, 2012):

- Diccionarios de tipo I: alumnos de primero y segundo grado de la enseñanza obligatoria. Son diccionarios adecuados para la fase inicial de alfabetización.

- Diccionarios de tipo II: alumnos de tercero y cuarto grado, que corresponde a la enseñanza media. Son diccionarios útiles para la fase de consolidación del dominio de la escritura.

- Diccionario de tipo III: alumnos de quinto a octavo, que corresponden a los últimos cursos de la enseñanza obligatoria. ${ }^{4}$

En relación con el número de entradas, se estableció que los diccionarios de tipo I debían contener entre 1.000 y 3.000 entradas; los diccionarios de tipo II, entre 3.500 y 10.000 entradas, y los de tipo III, entre 19.000 y 35.000. Esta comisión propuso también una serie de criterios (según la letra, la inclusión de dibujos, la redacción de la definición, etc.) que debería cumplir cada tipología para responder a las necesidades pedagógicas (Carvalho, 2011). Por ejemplo, se llegó a la conclusión de que las nomenclaturas de los diccionarios de tipo I o II no podían ser versiones reducidas de diccionarios generales para adultos o de diccionarios de tipo III. La microestructura de los diccionarios de tipo I debía ser muy didáctica, de modo que el alumno entendiera la definición y, por lo tanto, las palabras se tenían que definir con palabras fáciles. La comisión, además, recomendó el uso de definiciones oracionales 5 para seleccionar los diccionarios adecuados para estas primeras edades (Cowie, 2000).

En el marco de la lexicografía escolar en catalán, solo existen diccionarios escolares generales de lengua destinados a niños y niñas a partir de 9 años, es decir son de tipo II y sobre todo de tipo III. Los diccionarios más significativos de la lexicografía escolar catalana reciente son los siete siguientes:

2. En el ámbito anglosajón o francófono, por citar dos ejemplos próximos, los niños cuentan con un gran repertorio de diccionarios adecuados para diversas edades. Los títulos son reveladores: Oxford Very First Dictionary, Oxford First Dictionary, Mon premier Larousse: le dictionnaire de 4-7 ans, Mon premier dictionnaire: le Robert Benjamin 6-8 ans, etc.; y también especializados, como: S Maths Dictionary for kids (Eather, 2013) o Science Dictionary for kids (Westphal, 2009).

3. De hecho, la inclusión de diccionarios para la enseñanza fundamental en las escuelas públicas nacionales de Brasil se produjo en 2001 a través de minidiccionarios de uso escolar, pero no fue hasta el PNLD de 2006 cuando se avanzó significativamente con la creación de una comisión específica que analizó y seleccionó diccionarios y estableció unas directrices para seleccionar o elaborar diccionarios. Estas directrices se pueden consultar en la página web siguiente: http://portal.mec.gov.br. 4. En 2012, esta misma comisión amplió la tipología de diccionarios portugueses didácticos y estableció un diccionario tipo IV para los alumnos de los dos últimos cursos de la enseñanza obligatoria.

5. En un momento en el que la mayoría de los diccionarios que analizó esta comisión presentaban un patrón de definición clásico, estableciendo una equivalencia entre la definición y la palabra-entrada que refleja el Principio de sustituibilidad (Landau, 1984; Weinreich, 1984). 
- BARCANOVA (2009). Diccionari Barcanova de la llengua escolar. Según su prólogo está destinado a alumnos de primaria y contiene 20.000 entradas. Se trataría de un diccionario de tipo III.

- CRUÏLLA (2004). Diccionari bàsic de la llengua catalana. Los destinatarios son los alumnos de primaria y los de los primeros cursos de ESO. También ofrece unas 20.000 entradas. Sería un diccionario de tipo III.

- ENCICLOPÈDIA CATALANA (1995) DIDAC. Diccionari de català. En su prólogo no se precisa la edad de los niños y las nińas a los que va dirigido el diccionario, solo se especifica que está destinado a la enseñanza primaria. No obstante, el hecho de que contenga 15.282 entradas nos permite decir que está destinado a los dos últimos cursos de primaria. Se trataría pues de un diccionario de lengua general a medio camino entre tipo II-III.

- EUMO Editorial (1990). Primer diccionari. Se recomienda para niños y niñas de 8 a 12 años. Contiene 9.246 entradas. Sería pues un diccionario de lengua general tipo II.

- ONDA (2004). Diccionari junior nou. Ofrece 26.000 entradas y está destinado a educación primaria y al primer ciclo de la ESO. Es un diccionario de tipo III.

- SANTILLANA Grup Promotor (2012). Nou Diccionari escolar de la llengua catalana. Contiene 24.000 entradas y no especifica para qué edades es adecuado. Es por lo tanto un diccionario de tipo III.

- VOX (2009). Diccionari escolar de la llengua catalana. Contiene 19.000 entradas. Es también un diccionario de tipo III.

Así pues, todos los diccionarios escolares en lengua catalana, siguiendo el criterio del número de entradas, son de tipo III, excepto uno -Primer diccionari de EUMO-, que sería de tipo II. Además, todos son de lengua general, puesto que la mayoría de la terminología académica que incorporan se encuentra al mismo nivel que el léxico general de la lengua, sin ninguna marca temática orientativa.

En la lexicografía española, el panorama no es muy diferente. Castillo y García Platero (2003) documentan tres obras lexicográficas de tipo I, pero remarcan que «no se trata de diccionarios propiamente dichos ya que las palabras no tienen definición, pues son las ilustraciones que contiene las que remiten a los distintos vocablos». Se trata entonces de imaginarios o de diccionarios en imágenes, como: Imaginario. Diccionario en imágenes para niños, publicado por SM en 1992; Mis primeras 1000 palabras, de Ferrándiz, publicado por Libsa en 1998, o el Pictoriccionario. Diccionario en imágenes, de Santillana, 1995. Este último con algunas palabras que presentan una definición sencilla.

En relación con los diccionarios de ciencias -o de matemáticas- en catalán, el vacío es mucho mayor, ya que no conocemos la existencia de ningún diccionario de estas características para alumnos de primaria y, menos aún, para alumnos específicamente de los dos primeros cursos de primaria. Hemos referenciado un vocabulario de matemáticas en línea (http://www.xtec.cat/ sgracia $5 /$ ), elaborado por Sergio Gracia, que forma parte de su memoria de licencia para realizar un plan de acogida de estudiantes extranjeros. Este vocabulario se basa en la lista de palabras que acompaña el currículum oficial de primaria (en el área de matemáticas) del Reino Unido. Este recurso, que tiene algunos problemas lexicográficos y de operatividad, ofrece una primera aproximación al vocabulario que se usa en las aulas en tres niveles, representados por tres colores distintos. La lengua castellana también adolece de este tipo de obras. No hemos encontrado diccionarios actuales de ciencia elaborados en la Península Ibérica que sean independientes y cuyo público destinatario sea el alumnado de las primeras etapas de primaria. En el Instituto de la Sordera de Chile, en cambio, elaboraron una serie de diccionarios escolares visuales y electrónicos sobre diferentes ámbitos científicos para niños (http://www.institutodelasordera.cl/enlaces/webh/). A pesar de los problemas técnicos de accesibilidad del portal, esta experiencia representa una primera aproximación. 
En otras tradiciones escolares, en cambio, existen proyectos lexicográficos interesantes en papel, pero también recursos en soporte digital accesibles a través de internet. Por citar solo algunas referencias, destacamos: Science Dictionary for kids (grades 4-9), de Laurie E. Weestphal (2009); Scholastic Science Dictionary, de Burger y Brunner (2000); Math Dictionary for kids, de Fitzgerals (2011); First Illustrated Science Dictionary, de Khan (2013), y Junior Science Dictionary (Maths and Science Dictionaries), de Glover (2011). Y, en especial, hay que destacar la labor de la editorial Oxford, que en los últimos años ha publicado diferentes diccionarios escolares clasificados según la materia y la edad. Por ejemplo, para los niños de 5 a 8 ańos: Oxford First Illustrated Science Dictionary (2001), Oxford First Illustrated Maths Dictionary (2001), Oxford First Science Dictionary (2001) y Oxford First Maths Dictionary (2001); para los niños de 9 a 11 años: Oxford Primary Illustrated Science Dictionary (2003) y Oxford First Primary Maths Dictionary (2003), y referidos a la enseñanza secundaria: Oxford Student's Science Dictionary (2006). ${ }^{6}$

Por ejemplo, el Oxford First Illustrated Science Dictionary es un diccionario de 200 palabras básicas de la ciencia con definiciones e ilustraciones para niños de 4 a 6 años, tal y como se puede observar en la imagen siguiente:

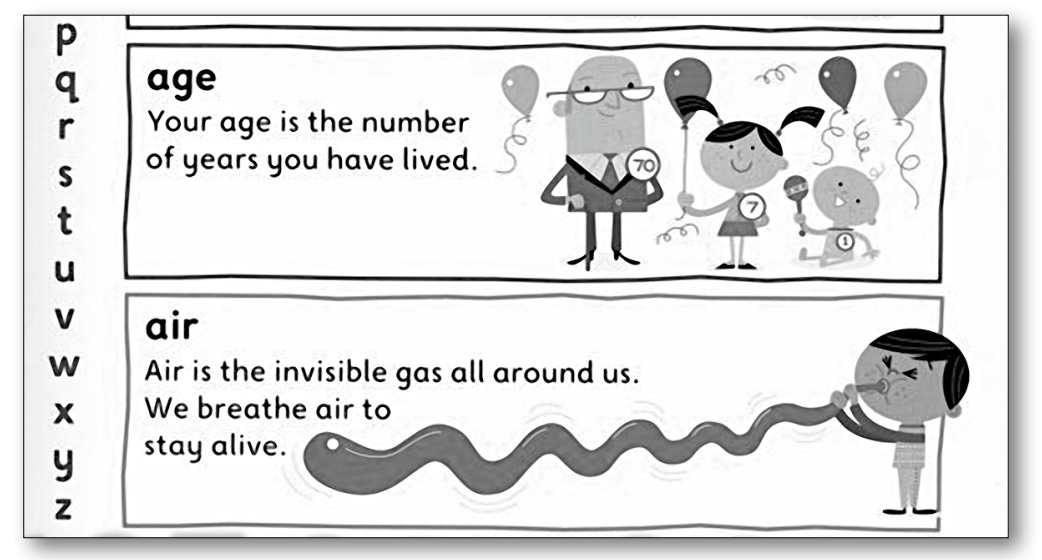

Imagen 1. Entradas age y air del Oxford First Illustrated Science Dictionary.

En consecuencia, es un axioma lexicográfico que no todos los diccionarios escolares son útiles para todos los usuarios, de la misma manera que es falso pensar que con un buen diccionario escolar es suficiente para acompañar toda la escolaridad obligatoria. Los niños necesitan un diccionario para cada edad o para cada etapa escolar, ya que se van produciendo cambios tanto en su capacidad cognitiva como en la comprensión. No sirve, pues, utilizar el mismo diccionario cuando los nińos tienen 7, 9 u 11 años. $Y$, aunque es cierto que los diccionarios escolares han evolucionado mucho en los últimos veinte años (Pérez Lagos, 1998), todavía no tenemos en nuestra tradición lexicográfica una variedad de diccionarios adecuados al primer ciclo de primaria y referentes a ámbitos especializados.

6. Existen también diversos portales que ofrecen diccionarios o vocabularios de ciencias en inglés como, por ejemplo, Math Dictionary for Kids, de Eather (http://www.amathsdictionaryforkids.com/), o Illustrated Mathematics Dictionary, de Pierce, que forma parte de un portal para aprender matemáticas: Maths is fun (http://www.mathsisfun.com/aboutmathsisfun.html). 


\section{OBJETIVOS}

El objetivo de este artículo es presentar la metodología que hemos utilizado para la elaboración de un diccionario escolar de ciencias destinado a niños y nińas del ciclo inicial de primaria.

Nuestra finalidad era elaborar un primer diccionario de palabras de ciencia adecuado a la primera etapa de la enseñanza obligatoria destinada a la alfabetización. En concreto, nos propusimos construir un diccionario de tipo I con los 100 términos más generales de la ciencia útil en el ciclo inicial de primaria, es decir, para nińos y niñas de 5 a 8 ańos. Este diccionario estaba plenamente justificado por el vacío lexicográfico existente, tanto en lo que concierne a la temática especializada como a los destinatarios de la obra.

\section{HIPÓTESIS}

En nuestro proyecto, partimos de la idea de que debíamos diseñar un diccionario de términos básicos de ciencia para niños y niñas de 6 a 9 años que respetara el principio de adecuación de la metodología terminográfica dentro del marco de la teoría comunicativa de la terminología (TCT) (Cabré, 1999). Según este principio, cada trabajo terminográfico concreto adopta una estrategia metodológica individualizada en función de unas necesidades terminológicas propias relacionadas con la actividad de los usuarios, el contexto, la temática, los objetivos y los elementos y recursos disponibles.

Uno de los principales problemas con los que se topan los niños es el significado de los términos que usan los maestros, los libros, las pruebas, los ejercicios sobre cuestiones científicas en un sentido muy amplio. Para comprender los textos -orales o escritos- sobre ciencias, deben entender con sus palabras (con sus esquemas cognitivos) qué significan las palabras de ciencia, a qué se refieren y cómo se usan. En consecuencia, nuestra hipótesis de trabajo fue la siguiente: la máxima adecuación cognitiva y expresiva de un diccionario a su público final se puede conseguir a partir del conocimiento acumulativo positivo de un colectivo representativo del conjunto de usuarios que lo utilizará. Esto significaba partir de un corpus de representación del conocimiento de los niños. Los niños expresan básicamente lo que saben con palabras y con dibujos. Por ese motivo, creamos un corpus de definiciones y dibujos de niños a partir del cual elaboraríamos el diccionario. Planteamos, pues, utilizar una metodología colaborativa y acumulativa que partiera del conocimiento científico de los niños y las nińas.

\section{PLANTEAMIENTO DEL PROBLEMA: EL PRINCIPIO DE ADECUACIÓN}

El principio de adecuación es uno de los principios vertebradores de la metodología de trabajo de la lexicografía especializada o de la terminografía en el marco de la TCT de M. Teresa Cabré (1999). Según esta autora, las aplicaciones terminológicas -como lo son los diccionarios especializados-deben adecuarse a las necesidades y al contexto social y lingüístico de las personas a quienes van destinadas. En las buenas prácticas terminográficas, la adecuación del trabajo terminológico a las condiciones específicas de cada situación es indispensable para asegurar el éxito de un diccionario, entendiendo en este caso como éxito la posibilidad de su uso como herramienta habitual de consulta.

Los productos terminográficos o de lexicografía especializada pueden tener características muy variadas y poder así servir a finalidades distintas. Producir un glosario, comenta Cabré, presupone analizar previamente la situación y características del proyecto que se va a producir y a continuación diseñarlo y planificarlo de acuerdo con estas características. Cabré concreta que esta manera de actuar se traduce en la aplicación del principio de adecuación. Según este, el trabajo terminológico aplicado 
no es monolítico sino variado en función de las circunstancias temáticas, sociolingüísticas, funcionales y contextuales en las que va a desarrollarse (Cabré, 1999).

En el campo de la lexicografía general, autores como Bergenholtz y Tarp (1990 y 2003), a través de su teoría moderna de las funciones lexicográficas, o Alvar (1982) ya habían reclamado también que los diccionarios debían adecuarse más a las diversas necesidades que contemplan los usuarios:

Las editoriales no se han planteado con rigor una programación de publicación de diccionarios que cubran el mayor número posible de necesidades, quedando al descubierto, o con pocos elementos entre los que escoger, áreas que deberían estar mejor atendidas. A pesar de lo que pueda parecer el del diccionario es un mercado de baja competencia comercial [...] Y es bien sabido que, a falta de competencia, la calidad no aumenta (Alvar, 1982: 41).

Dictionaries are considered utility products that are made in order to satisfy certain human needs. Consequently, all theoretical and practical considerations must be based upon a determination of these needs, i.e. what is needed to solve the set of specific problems that pop up for a specific group of users with specific characteristics in specific user situations (Bergenholtz y Tarp, 2003: 172).

La idea de adecuación entre diccionario y objetivo de enseñanza también es un principio básico de la lexicografía pedagógica (Hernández, 1989). En lexicografía escolar diversos autores habían discutido la necesidad de adaptar los diccionarios escolares a sus usuarios (Jakson, 2002) y esto conllevó una gran revolución en la industria editorial lexicográfica escolar que, en el marco de la lexicografía catalana, llegó a finales de la década de los ochenta y que supuso que los diccionarios para la escuela no fueran simples reducciones de los diccionarios de lengua general, tal y como había denunciado Hernández (1989). En un plano internacional, sin embargo,fue sobre todo Sinclair quien lideró este cambio de paradigma con la elaboración del diccionario COBUILD (Moon, 2007), una propuesta de diccionario de aprendizaje que implicó un cambio de metodología relevante.

En el panorama de la lexicografía española, en la última década del siglo pasado, autores como Hernández (1989) y Martínez Marín (1991) señalaban una serie de defectos de los diccionarios escolares, que Azorín (1998: 78) resume en los siguientes puntos:

- La insuficiente o nula delimitación tanto de la finalidad didáctica que persigue la obra como del colectivo al que se dirige.

- La inadecuada selección del corpus registrado.

- La falta de precisión o excesiva generalidad en la formulación de las definiciones, que suelen presentar, en ocasiones, casos de circularidad o de pistas perdidas.

- Ausencia de un criterio uniforme en la ordenación de las acepciones, así como en la ubicación de los elementos fraseológicos.

- Graves insuficiencias en el apartado de la información gramatical.

- Escasa o nula presencia de ejemplos que orienten en el uso y favorezcan, por tanto, la función codificadora del diccionario.

- Escasa atención a los aspectos pragmáticos y sociolingüísticos del léxico.

Maldonado (1998: 27) completó esta lista refiriéndose a lo que considera como tres puntos flacos: la escasez de obras destinadas a los escolares, la dificultad del docente para motivar a los alumnos y la falsa idea de que todos los diccionarios son iguales. Aunque algunas de estas deficiencias se siguen arrastrado en obras contemporáneas, es cierto que hoy día disponemos de diccionarios escolares mucho más adecuados a las necesidades de los niños y las nińas, con relación tanto a la macroestructura (sobre todo en la selección de la nomenclatura) como a la microestructura (aspectos gramaticales, definición, ejemplos) (Castillo y García Platero, 2003). Actualmente, la mayoría de diccionarios escolares simulan las explicaciones del escolar y potencian la definición oracional. Por ejemplo, en el contexto 
catalán, los primeros diccionarios elaborados con esta metodología fueron el Primer Diccionari (1990), de EUMO, dirigido por prestigiosos profesores de la Escuela de Magisterio de la Universidad de Vic, y el Diccionari de català DIDAC (1995), de Enciclopèdia Catalana, bajo la dirección de la lexicógrafa M. Teresa Cabré. No obstante, la definición de este tipo de diccionarios no deja de ser una simulación, ya que sus definiciones imitan las narrativas explicativas de los niños pero no son reales. ${ }^{7}$

Diversos trabajos, entre ellos destacamos los realizados por Pérez Lagos (1998), Ávila Martín (2000), Prado Aragonés (2000) o los del Grupo de Lexicografía y Lingüística de Corpus dirigidos por Azorín (1998) (2007), demuestran que a pesar del «indudable avance experimentado por la lexicografía escolar, los diccionarios [castellanos -y también los catalanes-] siguen presentando, a juicio de sus usuarios habituales, importantes deficiencias, principalmente: en cuanto a la cantidad y calidad de las voces que registran; en el tratamiento de la fraseología; en la formulación de las definiciones; en la ejemplificación; y en las ilustraciones».

Esta inadecuación a las necesidades del usuario lleva a una infrautilización del diccionario como herramienta didáctica, aspecto este que remarcan los más ilustres teóricos de la lexicografía y de la didáctica (Rey-Debove, 1971). A nuestro entender, el diccionario se introduce tarde en las aulas, y los niños y las niñas no acaban de conectar con él. En algunos casos porque no son adecuados a las necesidades de una edad concreta, en otros, en cambio, porque no se les otorga la importancia debida.

\section{METODOLOGÍA}

Con la finalidad de ser lo más adecuados posible a las necesidades de uso de los niños y las niñas de primero y segundo de primaria, nuestra estrategia fue trabajar de manera colaborativa entre escuela y universidad para así recopilar un corpus de definiciones y dibujos realizados por niños y nińas.

La utilización de corpus de textos escolares ya se había mostrado eficiente en la lexicografía escolar. Desde el año 2011, un equipo de Oxford University Press, junto con el Lexical Computing Departament de Oxford University, está llevando a cabo experiencias muy novedosas en la construcción de diccionarios para niños a partir de corpus reales escritos por ellos mismos. Así, un subcorpus del Oxford Children's Corpus (OCC) -el Beebox- está constituido por escritos de unas 500 palabras elaboradas por niños y niñas distribuidos en tres niveles de edades (5-8 años, 9-10 años y más de 11 años) sobre un tema libre (Wild et al., 2011 y 2013). La BBC Radio 2 organiza anualmente un concurso de historias cortas escritas por niños de 5 a 13 años. El subcorpus de textos de niños procede de los escritos del concurso que organizó la BBC en 2011. De manera que, a principios de 2013, el OCC incluía un subcorpus de 73.875 historias (2/12 partes de las historias correspondían a niños entre 5 y 8 años, 5/12 a niños entre 9 y 10 años y las 5/12 partes restantes a niños entre 11 y 13 años) que respondían a unas 32 millones de ocurrencias. Los temas de las historias son muy diversos y generales: acerca de personas (madre, abuelo, hermano, maestro, etc.), animales, lugares, fiestas, con tramas que dan miedo, etc. Desde 2006, los lexicógrafos del Oxford University Press utilizan este tipo de corpus para complementar la información lexicográfica de sus diccionarios para niños.

Nuestro corpus, en cambio, se constituyó con definiciones-explicaciones de palabras y dibujos. Para recopilar este material, trabajamos durante tres cursos escolares directamente en la escuela: 500 niños y niñas definieron a través de explicaciones (breves narrativas) y de dibujos cerca de 100 términos básicos de las ciencias. El trabajo de recopilación de los datos se realizó siempre en el aula durante los cursos escolares que van desde 2009 a 2012. Participaron tres escuelas distintas de Girona, Sabadell y Pineda de Mar. El trabajo se llevó a cabo principalmente en las dos primeras escuelas, ya que compar-

7. Podríamos establecer un paralelismo con la diferencia que existe entre un buen ejemplo y un contexto de uso real. 
tían características sociolingüísticas similares, y tan solo en un 10\% del diccionario participó la tercera escuela, siendo utilizada como escuela de control durante el tercer año de trabajo.

Con relación al nivel lingüístico de los participantes, se priorizó el criterio de familiaridad y fluidez con la lengua catalana. Por este motivo se seleccionaron dos escuelas de un entorno social catalanohablante, conocidas por su espíritu innovador y experimental tanto respecto a las ciencias como a la lengua. El 85\% de sus alumnos tienen la lengua catalana como lengua materna, siendo la lengua habitual de relación entre ellos el catalán. El segundo criterio armonizador fue el nivel social de las familias. Son niños que proceden de familias con un nivel cultural y económico medio-alto (alrededor del $80 \%$ de los padres y madres de estos alumnos tienen estudios superiores). Optamos por este tipo de escuelas por dos motivos principales: primero, porque necesitábamos a niños y niñas que en el primer curso de primaria pudiesen expresarse por escrito y, sobre todo, porque a partir del conocimiento, científicamente adecuado, acumulativo de los alumnos teníamos que construir una definición completa y adecuada que fuera útil al resto de población de su misma edad. Para ello, era más adecuado partir de niños y niñas que, a priori, contaban con más conocimientos y con un entorno familiar más cultivado, ya que es sabido que en estas edades las concepciones o representaciones están fuertemente influenciadas por el nivel de conocimiento e historia contextual (Marín, 2003). Así pues, no podíamos trabajar con niños y niñas que elaborasen definiciones excesivamente simples desde el punto de vista cognitivo, o bien lexicalmente pobres, ya que entonces aquellas no servirían para todos o, incluso, necesitaríamos muchos más inputs. No obstante, en el tercer año del proyecto, incorporamos a una escuela de características opuestas que nos sirvió de escuela control. En concreto con una clase de segundo curso de primaria en la que los padres y madres de un 35,6\% de los alumnos eran inmigrados, un $22 \%$ usaban la lengua catalana como principal y solo el $2 \%$ de los padres tenían estudios superiores.

El trabajo se realizó principalmente en el contexto del aula propia de los alumnos, con el objetivo de evitar el máximo de interferencias externas. No obstante, se utilizaron también otros espacios escolares: el aula de lengua, la de proyectos y el aula de plástica. De la misma manera, la mayor parte del trabajo se realizó en una franja horaria periódica y sistematizada reservada siempre al diccionario (una hora semanal).

El lemario fue seleccionado después de aplicar diferentes criterios: a) primero se seleccionaron los términos más básicos que se incluían en diccionarios de tipo II y tipo III y en libros de texto; $b$ ) después se restringió la primera selección y se comprobó que todas las palabras estuvieran incluidas en la lista de términos de Tennessee correspondiente a la edad escolar de 6-8 años (Tennessee Academic Vocabulary del Tennessee Departament of Education, 2009), y c) finalmente la lista fue validada por maestros de primaria de tres centros distintos. Una muestra de las palabras seleccionadas es la siguiente: agua, aire, balanza, calor, cerebro, experimento, fuego, idea, luna, lupa, memoria, muerte, neurona, ordenador, problema, resta, sonido, termómetro, triángulo, universo, etc. Algunas son palabras que se utilizan en la lengua común y que forman parte del vocabulario base de la ciencia.

El resultado fue un corpus de dibujos y de narraciones explicativas reales de niños y niñas de primer y segundo curso de primaria (5-9 años): el IULATERM Children's Corpus (ICC). El ICC está formado por 3.550 definiciones de palabras concretas relacionadas con la ciencia, que corresponden a 68.639 ocurrencias, y por 3.575 dibujos de esos conceptos científicos. Las definiciones son breves narraciones que explican el significado de esas palabras. Los niños trabajaron en papel y toda la información se digitalizó, de manera que ahora es accesible a través del portal abierto El Microscopio en la siguiente dirección web: http://defciencia.iula.upf.edu/Microscopi (Estopà y Cornudella, 2013). ${ }^{8}$

El ICC se usó para elaborar cada una de las definiciones del diccionario. Para construir la definición-explicación final se usó una estrategia de acumulación de información positiva. Así, se seleccionó

8. Solo se corrigieron los errores de ortografía o de gramática. 
toda la información positiva desde el punto de vista científico de las definiciones del ICC, respetando las palabras usadas por los escolares en sus escritos. Para ello, se desechó cualquier información tóxi$c a$, imaginaria o perteneciente a un saber cultural o contextual. En la última etapa se llevó a cabo un control del conocimiento por parte de especialistas (biólogos, médicos, físicos, ingenieros). De esta manera, diversos científicos aseguraron la autenticidad de la información seleccionada desde el punto de vista científico.

Se elaboraron las definiciones siguiendo los patrones más recurrentes que usaban los escolares. Se incluyeron en todas las definiciones ejemplos y contextualizaciones que habían mencionado los niños. Este tipo de información contribuye enormemente a la comprensión del término. Los ejemplos nunca se inventaron o manipularon; son los que propusieron los niños y niñas en sus narraciones. Por ejemplo, en la palabra huevo se dice:

... Los huevos pueden ser de diferente tamaño, por ejemplo, los del avestruz son más grandes que los de la gallina [...] Nosotros solemos comer huevos de gallina y usamos este alimento para hacer, por ejemplo, una tortilla, un huevo frito o un huevo duro o también cuando queremos hacer una torta, un pastel o magdalenas.

Y en la palabra cerebro se dice: «El cerebro es una parte del cuerpo humano que está dentro del cráneo y que sirve para pensar y también para estudiar, leer, escuchar, hablar, andar, trabajar, elegir...».

Con relación a la inclusión de acepciones, se usó el mismo criterio: se incluyeron solo las acepciones que los niños detectaron. En el momento en el que un niño (aunque fuera solo uno) definía una palabra con una acepción nueva la incluimos en el diccionario. Es el caso, por ejemplo, de acuario, termómetro, tierra, problema o planta. En cambio, otras veces los niños coincidían en destacar un significado único, a pesar de ser un término polisémico. Entonces, recogíamos solamente el significado atestiguado por los nińos, como sucedió, por ejemplo, con balanza, memoria o espacio.

Finalmente, también se consideró que los dibujos son un complemento muy potente de las narraciones breves, es decir, de las definiciones. Por un lado, permiten integrar a todos los niños, pues en el caso de que alguien no se supiera expresar escribiendo podía participar solo dibujando (lo que ocurrió en un $4 \%$ de los alumnos de primer curso de primaria). Por otro lado, los dibujos de niños muestran con detalle ciertos matices que no quedan reflejados en las explicaciones. Había términos extremadamente difíciles de representar: conceptos abstractos o no tangibles sin ayuda de algún instrumento. Resulta complejo dibujar términos como fuerza, aire o neurona; y, en cambio, eso no supuso un obstáculo. Sus representaciones reflejan la noción común que esconden estos términos en estas edades tan tempranas. ${ }^{9}$

\section{RESULTADOS: MI PRIMER DICCIONARIO DE CIENCIA, UN DICCIONARIO DE CIENCIAS COLABORATIVO ESCOLAR}

El Petit diccionari de ciència (Mi primer diccionario de ciencia) ${ }^{10}$ (PDC) es, pues, un diccionario de tipo I especializado, que contiene 100 términos básicos que corresponden a 126 acepciones distintas; 13 son unidades verbales y el resto son nombres. Está destinado a acompañar el aprendizaje de niños y niñas de primero, segundo y (en algunos casos) tercero de primaria. Cada una de las entradas incluye la categoría gramatical, una explicación y un dibujo.

9. Para el diccionario solo seleccionamos, por cuestiones de edición, un único dibujo. No obstante, puede consultarse una selección de dibujos a través de El Microscopio o en la página web del proyecto: http://defciencia.iula.upf.edu/index_eng. htm.

10. Publicado también en castellano por Publicacions de l'Abadia de Montserrat (2013): Mi primer diccionario de ciencia. 
La explicación del término es una narración de cómo el niño define esta palabra, de cómo la entiende, de qué significa para él, de cómo la usa. Es una definición lexicográficamente poco canónica, puesto que siempre es multioracional, situacional y pragmática. La información está ordenada siguiendo la importancia que los niños han dado a las características semánticas en sus definiciones. Las definiciones del PDC muchas veces siguen patrones como "X es un» (normalmente seguido de un hiperónimo y no siempre del mismo nivel) o «Cuando...» (normalmente para explicar el significado de un verbo). Veamos algunos ejemplos de cómo se inician las definiciones en el diccionario final:

aire

El aire es transparente e incoloro...

\section{brújula}

Una brújula es un objeto parecido a un reloj...

cientifico/cientifica

Un científico o una científica es una persona...

contar

Cuando contamos decimos cuántas cosas hay...

bielo

El hielo es agua congelada...

número

Un número se usa para contar...

tiempo

El tiempo es lo que pasa entre dos hechos o también lo que duran las cosas...

Las relaciones de función y de parte-todo son las más recurrentes entre sus narraciones. Como ya es sabido, a los nińos les interesa más para qué sirven las cosas que lo que son; de manera que la relación funcional es más relevante que la ontológica. Las explicaciones de los niños además están estrechamente relacionadas con su contexto experimental, por eso es tan importante que las definiciones recurran a los ejemplos y a las situaciones pragmático-comunicativas. Así, hemos optado por incluir el máximo número de contextos que acercaran al niño a su realidad más próxima. Estas decisiones metodológicas están avaladas por especialistas en didáctica de la ciencia, como Driver (1983), De Posada (1996) o Marín (2003), que sostienen que «en el sujeto de la etapa infantil son más relevantes las interacciones que dan lugar a una estructura de carácter procedimental e implícitamente muy ligada a lo efectivo» (Marín, 2003: 80).

A continuación, reproducimos una entrada del diccionario: estrella. Si la analizamos, constatamos que en realidad no se dice qué es una estrella (que es la esencia de la definición clásica), sino cómo es, dónde se puede ver, de qué está constituida, cuántas hay y ejemplos de estrellas. Además, en esta definición es muy interesante el detalle discursivo «... aunque no parezca una estrella como las otras», porque refleja una complicidad cognitiva con el niño, dado que muchos niños se sorprenden de que el Sol sea una estrella, pues la vemos de día y no de noche, pareciendo a nuestros ojos mucho mayor, más brillante y más potente que las demás que solemos contemplar por la noche. 


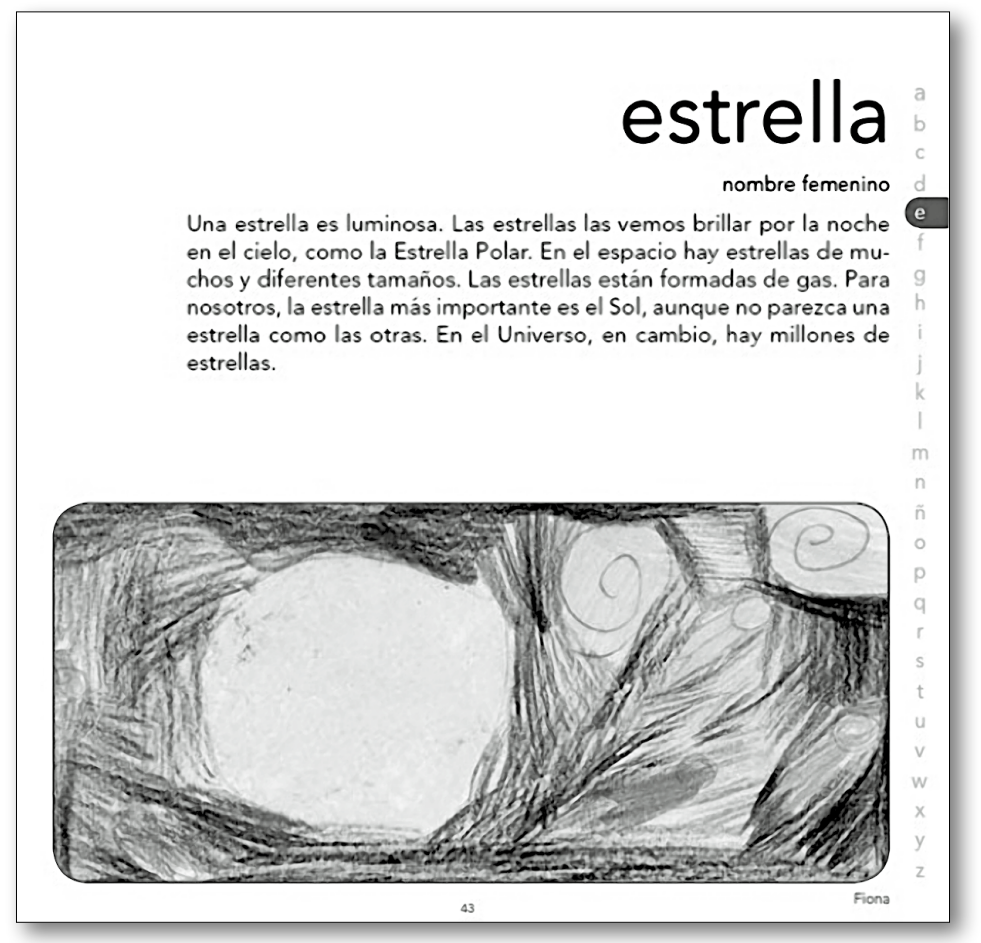

Imagen 2. Entrada estrella de Mi primer diccionario de ciencia.

\section{EVALUACIÓN Y VALORACIÓN DEL RESULTADO}

Sabemos por las comunicaciones realizadas con los niños y niñas que han usado el diccionario que lo entienden, les gusta y los dibujos les fascinan. También diversos maestros nos han comentado que el diccionario les parece muy adecuado y útil al no disponer de una obra en catalán o en castellano parecida. No obstante, hemos realizado una prueba experimental para ratificar estas primeras impresiones, ya que de lo contrario corrían el riesgo de ser excesivamente subjetivas. Con este objetivo diseñamos una prueba de compresión: queríamos comprobar si, para los niños y niñas de 6 a 8 años, las explicaciones elaboradas para Mi primer diccionario de ciencia son más adecuadas que las definiciones que incluyen otros diccionarios escolares existentes en la lexicografía catalana.

El colectivo que realizó la prueba comprendía a niños y niñas de primer curso (70 alumnos, 3 aulas), segundo (91 alumnos, 4 aulas) y tercero ( 25 alumnos, 1 aula) de primaria de tres escuelas de Pineda de Mar, Banyoles y Santa Susanna, respectivamente. Eran niños y nińas que no habían participado en la elaboración del diccionario. El nivel socioecómico y sociolingüístico de los niños de estas escuelas es variado y creemos que puede ser más o menos significativo de un porcentaje elevado de niños. La escuela de Pineda de Mar-como referimos anteriormente- es una escuela con una alta proporción de niños de padres extranjeros, que no tienen el catalán como lengua materna y con unos niveles socioeconómico y sociocultural bajos. La escuela de Banyoles es una escuela con un $25 \%$ de inmigración, con la mayoría de los padres catalanohablantes, con una situación socioeconómica media y con un $40 \%$ de padres con estudios superiores. La escuela Montagut tiene un porcentaje de inmigración bajísimo (5\%), el 60\% de los padres no tienen el catalán como lengua materna, el nivel socioeconómico de los padres es medio-alto y el nivel sociocultural es también medio-alto (el 70\% de los niños tienen uno de los padres con estudios superiores). Las tres escuelas están situadas en poblaciones con muchos servicios y cercanas a la capital de provincia. 
Seleccionamos tres términos: agua, cerebro y telescopio. Luego extrajimos las definiciones de estos términos de cuatro diccionarios escolares: Eumo (tipo II), Santillana (tipo III), Didac (tipo III) y PDC (tipo I). Las mezclamos y las ofrecimos en cada pregunta con un orden distinto. Les pedimos a los participantes en la prueba que pusieran una cruz verde en la explicación que entendían mejor y una cruz roja en la que entendían peor o que no entendían. También les pedimos que en todas las definiciones marcaran en rojo las palabras que no comprendían. La prueba fue pasada por sus maestros en su aula habitual. Reproducimos las preguntas de la prueba de comprensión:

Què és un cervell? ('¿Qué es un cerebro?’)

A. Part més gran de l'encèfal dels vertebrats, situada a la zona del davant i de dalt del crani. ('Parte más grande del encéfalo de los vertebrados, situada en la zona de delante y de arriba del cráneo').

B. El cervell és una part del cos humà que és dins del crani i que serveix per a pensar i també per a estudiar, llegir, escoltar, parlar, caminar, treballar, escollir... El cervell és tou, arrodonit i està format per neurones. Hi ha animals que també tenen cervell, però és diferent del de les persones. Si no tinguéssim cervell, no seríem persones. ('El cerebro es una parte del cuerpo humano que está dentro del cráneo y que sirve para pensar y también para estudiar, leer, escuchar, hablar, andar, trabajar, elegir... El cerebro es blando, redondeado y está formado por neuronas. Hay animales que también tienen cerebro, pero es diferente del de las personas. Si no tuviéramos cerebro no seríamos personas').

C. El cervell és un òrgan tou, de forma arrodonida i amb molt plecs que hi ha dins del cap de les persones. Molts animals també tenen cervell. És una part de l'encèfal. El pensament i els moviments voluntaris depenen del cervell. ('El cerebro es un órgano blando, de forma redondeada y con muchos pliegues que está dentro de la cabeza de las personas. Muchos animales tienen cerebro. Es una parte del encéfalo. El pensamiento y los movimientos voluntarios dependen del cerebro').

D. Òrgan de la intel-ligència que es troba a l'interior del cap de l'home i dels animals. ('Órgano de la inteligencia que se encuentra en el interior de la cabeza del hombre y de los animales').

La primera (A) procede del diccionario Santillana, la segunda (B) del PDC, la tercera (C) del DIDAC y la última (D) del diccionario d'EUMO.

Què és l’aigua? ('¿Qué es el agua?')

A. L'aigua és un líquid incolor que no té gust ni fa olor. L'aigua és formada d'oxigen i hidrogen. És un gran dissolvent. Es torna sòlida a $0{ }^{\circ} \mathrm{C}$ i forma el gel, i bull a $100{ }^{\circ} \mathrm{C}$. La seva fórmula és: $\mathrm{H}_{2} \mathrm{O}$. ('El agua es un líquido incoloro que no tiene gusto ni huele. El agua está formada por oxígeno e hidrógeno. Es un gran disolvente. Se vuelve sólida a $0{ }^{\circ} \mathrm{C}$ y forma el hielo, y hierve a $100{ }^{\circ} \mathrm{C}$. Su fórmula es: $\left.\mathrm{H}_{2} \mathrm{O}^{\prime}\right)$.

B. Líquid format per hidrogen i oxigen, no té color, olor ni sabor. I es troba a la natura en els rius, mars i llacs. ('Líquido formado por hidrógeno y oxígeno, no tiene color, olor ni sabor. Y se encuentra en la naturaleza en los ríos, mares y lagos').

C. Líquid que hi ha als rius, als llacs i al mar: les aigües del riu baixaven brutes. ('Líquido que hay en los ríos, en los lagos y en el mar: Las aguas del río bajaban sucias').

D. L'aigua és un líquid transparent que podem beure i que també podem fer servir per a rentar-nos o regar les plantes. L'aigua de mar és salada i l'aigua de riu o de pluja, no. Les persones, els animals i les plantes necessitem aigua per a viure. ('El agua es un líquido transparente que podemos beber y que también podemos usar para lavarnos o regar las plantas. El agua del mar es salada y el agua del río o de la lluvia, no. Las personas, los animales y las plantas necesitamos agua para vivir'). 
La primera (A) procede del diccionario DIDAC, la segunda (B) del de Santillana, la tercera (C) del diccionario de EUMO y la última (D) del PDC.

Què és un telescopi? ('¿Qué es un telescopio?’)

A. Instrument que serveix per observar les estrelles, els planetes i els satèl-lits. ('Instrumento que sirve para observar las estrellas, los planetas y los satélites').

B. Un telescopi és un aparell òptic per a mirar objectes que estan molt lluny, sobretot les estrelles i altres cossos que hi ha la cel. Alguns telescopis són molt grans i estan tancats dins d'un edifici amb una cúpula que s'obre quan es vol mirar el cel. ('Un telescopio es un aparato óptico para mirar objetos que están muy lejos, sobre todo las estrellas y otros cuerpos que hay en el cielo. Algunos telescopios son muy grandes y están encerrados dentro de un edificio con una cúpula que se abre cuando se quiere mirar el cielo').

C. Instrument òptic que s'utilitza per observar objectes llunyans, per exemple els estels. ('Instrumento óptico que se utiliza para observar objetos lejanos, por ejemplo las estrellas').

D. Un telescopi és un aparell que enfoca el que està molt lluny per veureu-ho millor, com ara la Lluna, les estrelles, les constel.lacions i els planetes. En astronomia s'utilitza el telescopi per a observar i investigar l'Univers. ('Un telescopio es un aparato que enfoca lo que está muy lejos para verlo mejor, como la Luna, las estrellas, las constelaciones y los planetas. En Astronomía se utiliza el telescopio para observar e investigar el Universo').

La primera (A) procede del diccionario de EUMO, la segunda (B) del DIDAC, la tercera (C) del diccionario Santillana y la última (D) del PDC.

Los resultados de las pruebas en los distintos niveles escolares se recogen a continuación en las tablas 1, 2 y 3 . Hemos indicado en cada caso el número absoluto de escolares que seleccionaron una opción, pero también el porcentaje para poder comparar las respuestas entre las distintas edades, dado que el número de participantes por curso escolar no es idéntico:

Tabla 1.

Resultados de las pruebas de los escolares de tres aulas de primero de primaria

\begin{tabular}{|c|c|c|c|c|c|c|c|c|c|}
\hline \multirow{2}{*}{$1 . .^{\circ}$} & \multicolumn{2}{|c|}{ SANTILLANA } & \multicolumn{2}{|c|}{ DIDAC } & \multicolumn{2}{c|}{ EUMO } & \multicolumn{2}{c|}{ PDC } & \multirow{2}{*}{ Total escolares } \\
\cline { 2 - 9 } & + & - & + & - & + & - & + & - & \\
\hline \multirow{2}{*}{ cerebro } & 1 & 43 & 3 & 21 & - & - & 66 & 6 & 70 \\
& $1,4 \%$ & $61,4 \%$ & $4,3 \%$ & $30 \%$ & & & $94,3 \%$ & $8,6 \%$ & $100 \%$ \\
\hline \multirow{2}{*}{ agua } & 3 & 11 & 5 & 58 & 2 & 1 & 60 & - & 70 \\
& $4,3 \%$ & $15,8 \%$ & $7,1 \%$ & $82,9 \%$ & $2,9 \%$ & $1,5 \%$ & $85,8 \%$ & & $100 \%$ \\
\hline telescopio & 2 & 17 & 4 & 40 & 15 & 9 & 49 & 4 & 70 \\
& $2,9 \%$ & $24,3 \%$ & $5,7 \%$ & $7,1 \%$ & $21,5 \%$ & $12,9 \%$ & $64,3 \%$ & $5,7 \%$ & $100 \%$ \\
\hline
\end{tabular}

Tabla 2.

Resultados de las pruebas de los escolares de cuatro aulas de segundo de primaria

\begin{tabular}{|c|c|c|c|c|c|c|c|c|c|}
\hline \multirow{2}{*}{$2 . .^{\circ}$} & \multicolumn{2}{|c|}{ SANTILLANA } & \multicolumn{2}{|c|}{ DIDAC } & \multicolumn{2}{c|}{ EUMO } & \multicolumn{2}{|c|}{ PDC } & \multirow{2}{*}{ Total escolares } \\
\cline { 2 - 9 } & + & - & + & - & + & - & + & - & \\
\hline \multirow{2}{*}{ cerebro } & 3 & 25 & 8 & 54 & 20 & 5 & 60 & 7 & 91 \\
& $3,3 \%$ & $27,5 \%$ & $8,8 \%$ & $59,3 \%$ & $22 \%$ & $5,5 \%$ & $65,9 \%$ & $7,7 \%$ & $100 \%$ \\
\hline \multirow{2}{*}{ agua } & 6 & 8 & 1 & 72 & 29 & 5 & 55 & 6 & 91 \\
& $6,6 \%$ & $8,8 \%$ & $1,1 \%$ & $79,1 \%$ & $31,9 \%$ & $5,5 \%$ & $60,4 \%$ & $6,6 \%$ & $100 \%$ \\
\hline \multirow{2}{*}{ telescopio } & 7 & 15 & 7 & 50 & 32 & 14 & 45 & 12 & 91 \\
& $7,7 \%$ & $16,5 \%$ & $7,7 \%$ & $54,9 \%$ & $35,2 \%$ & $15,4 \%$ & $49,5 \%$ & $13,2 \%$ & $100 \%$ \\
\hline
\end{tabular}


Tabla 3.

Resultados de las pruebas de los escolares de un aula de tercero de primaria

\begin{tabular}{|c|c|c|c|c|c|c|c|c|c|}
\hline \multirow{2}{*}{$3 .^{\circ}$} & \multicolumn{2}{|c|}{ SANTILLANA } & \multicolumn{2}{|c|}{ DIDAC } & \multicolumn{2}{|c|}{ EUMO } & \multicolumn{2}{|c|}{ PDC } & \multirow{2}{*}{ Total escolares } \\
\hline & + & - & + & - & + & - & + & - & \\
\hline cerebro & - & $\begin{array}{c}19 \\
76 \%\end{array}$ & $\begin{array}{c}5 \\
20 \%\end{array}$ & - & - & $\begin{array}{c}1 \\
4 \%\end{array}$ & $\begin{array}{c}25 \\
100 \%\end{array}$ & - & $\begin{array}{c}25 \\
100 \%\end{array}$ \\
\hline agua & $\begin{array}{c}3 \\
12 \%\end{array}$ & - & - & $\begin{array}{c}22 \\
92 \%\end{array}$ & $\begin{array}{c}7 \\
28 \%\end{array}$ & $\begin{array}{c}1 \\
4 \%\end{array}$ & $\begin{array}{c}15 \\
60 \%\end{array}$ & $\begin{array}{c}1 \\
4 \%\end{array}$ & $\begin{array}{c}25 \\
100 \%\end{array}$ \\
\hline telescopio & $\begin{array}{c}6 \\
24 \%\end{array}$ & $\begin{array}{c}4 \\
16 \%\end{array}$ & $\begin{array}{c}2 \\
8 \%\end{array}$ & $\begin{array}{c}17 \\
68 \%\end{array}$ & $\begin{array}{c}5 \\
20 \%\end{array}$ & $\begin{array}{c}3 \\
12 \%\end{array}$ & $\begin{array}{c}12 \\
48 \%\end{array}$ & $\begin{array}{c}1 \\
4 \%\end{array}$ & $\begin{array}{c}25 \\
100 \%\end{array}$ \\
\hline
\end{tabular}

En las tres tablas se observa que las definiciones que entienden la mayoría de los alumnos son las del PDC y las que menos entienden son las del diccionario DIDAC y las del diccionario Santillana. Este resultado no debería sorprendernos, puesto que el DIDAC y el Santillana son los dos diccionarios escolares con más entradas y que responderían a diccionarios tipo II-III y III, respectivamente. Pero, en cambio, sorprenden los resultados referentes al DIDAC porque de los tres diccionarios es el que explícitamente tiene la voluntad -como se lee en el prólogo- de elaborar definiciones oracionales e incluir ejemplos cercanos a los niños de primaria. La parte gráfica parece próxima a los niños de primaria de las primeras etapas, pero no siempre la información cognitiva, tampoco para los niños de tercero. Quizá uno de los parámetros que no se adecuó a las necesidades de los niños participantes es la terminología que se usó en las definiciones. Por eso es relevante -y lógico- constatar que las definiciones que rechazan porque nos las entienden son las que incluyen más palabras marcadas en rojo, es decir, que más palabras desconocen -constatando que se trata de unidades terminológicas- o las definiciones que incluyen símbolos y fórmulas. El 95\% de los niños y las niñas que han participado en la prueba marcan en rojo las siguientes palabras:

Cerebro:

SANTILLANA: encèfal ('encéfalo') y vertebrat ('vertebrado')

EUMO: òrgan ('órgano')

DIDAC: encèfal ('encéfalo'), òrgan ('órgano'), plecs ('pliegues'), pensament ('pensamiento')

PDC: neurones ('neuronas')

Agua:

SANTILLANA: hidrogen ('hidrógeno'), oxigen ('oxígeno')

EUMO: -

DIDAC: 0 oC, 100 oC, H2O, sòlida ('sólida'), formula ('fórmula'), hidrogen ('hidrógeno'), dissolvent ('disolvente')

PDC: -

Telescopio:

SANTILLANA: òptic ('óptico'), llunyà ('lejano')

EUMO: satèl-lit ('satélite')

DIDAC: òptic ('óptico'), cúpula ('cúpula'), cossos ('cuerpos')

PDC: -

Puntualmente, también algún alumno ha señalado las siguientes palabras como desconocidas o que dificultan su comprensión: 
- Agua: incolor ('incoloro'), líquid incolor ('líquido incoloro') (DIDAC).

- Telescopio: astronomia ('astronomía'), constel-lació ('constelación') (PDC).

Las palabras astronomía y constelación que el PDC usa en la definición de telescopio seguramente son el motivo por el que en esta definición, aunque sigue siendo la más comprensible, los nińos bajaron el nivel de comprensión (primero: 64,3\%, segundo: 49,5\%, tercero: 48\%) en relación con las otras palabras estudiadas.

En cambio, la comprensión de las definiciones parece que no tiene relación ni con el número de palabras usadas ni con la cantidad de oraciones que presenta la definición. Los datos nos muestran que los diccionarios que utilizan una explicación con más de una oración -DIDAC y PDC- son con relación al grado de comprensión escolar opuestos, tal como se refleja en la tabla 4:

Tabla 4.

Número de oraciones empleadas en cada definición

\begin{tabular}{|l|c|c|c|c|}
\hline \multicolumn{5}{|c|}{ Numero de oraciones } \\
\hline & SANTILLANA & EUMO & DIDAC & PDC \\
\hline cerebro & 1 & 1 & 4 & 4 \\
\hline agua & 1 & 1 & 5 & 5 \\
\hline telescopio & 1 & 1 & 2 & 2 \\
\hline
\end{tabular}

\section{CONSIDERACIONES FINALES Y LÍNEAS DE TRABAJO FUTURO}

La metodología acumulativa y colaborativa utilizada, basada en un corpus de definiciones de niños para construir el diccionario, se ha mostrado adecuada: primero construir, después deconstruir y, finalmente, volver a construir. Los niños elaboran individualmente sus definiciones, nosotros las deconstruimos todas y acumulamos el conocimiento, y, por último, seleccionamos la información científicamente pertinente. Las definiciones del PDC, un primer diccionario de ciencia para niños y niñas de 5 a 8 años, son, pues, explicaciones basadas en el saber acumulativo controlado de los niños y las niñas de esta edad. El test experimental ha ratificado que los niños de esta edad entienden mejor el PDC que otros diccionarios escolares existentes en la lexicografía catalana destinados - desde nuestro punto de vista- a un nivel escolar superior.

A partir de esta experiencia hemos creado el Club LEXIC (Estopà y Cornudella, 2013; Estopà, 2013), que permite alimentar el corpus de definiciones tanto en relación con las edades de los niños como con la cantidad de textos. Esta herramienta ya está a disposición de las escuelas desde el curso 2013-2014 y es de acceso abierto en la siguiente dirección web: http://defciencia.iula.upf.edu/ClubLexicografs. Se trata de una plataforma digital que permite trabajar en castellano y en catalán, aunque es fácilmente adaptable a otras lenguas. Debido a las leyes de protección y de propiedad intelectual de menores fue necesario crear dos perfiles de usuarios distintos: uno para aquellos niños y niñas que fueran a colaborar anónima y puntualmente en la elaboración de futuros diccionarios colaborativos, y un perfil con más funcionalidades para aquellos niños y niñas que fueran a colaborar de forma más constante, incentivándolos a formar parte del Club LEXIC. Además, ser miembro permite poder disponer de un material diacrónico y trabajar, en la escuela, a posteriori con estos datos, como si se tratara de un portafolios digital individualizado sobre léxico. 


\section{REFERENCIAS BIBLIOGRÁFICAS}

Alvar, M. (1982). Función del diccionario en la enseñanza de la lengua. Revista de Bachillerato, 22, pp. 49-53.

Azorín, D. (1998). ¿Para qué usan los diccionarios los escolares? Reflexiones a propósito de una encuesta. Actas del congreso Tendencias en la lexicografía española actual. El diccionario como objeto de estudio lingüistico y didáctico. Huelva: Universidad de Huelva, 2000, pp. 75100.

Azorín, D. (2000). Los diccionarios didácticos del español desde la perspectiva de sus destinatarios. Estudios de lingüistica, 14, pp. 19-44.

Azorín D. (2007). La investigación sobre el uso del diccionario en el ámbito escolar. En A. Negri e I.M. Alves (orgs.) (2007). As Ciências do léxico: lexicologia, lexicografia, terminologia. Campo Grande: Ed. UFMS.

Barcanova (2009). Diccionari Barcanova de la llengua escolar. Barcelona: Barcanova.

Bergenholtz, H. y Tarp, S. (2003). Two opposing theories: On H.E. Wiegand's recent discovery of lexicographic functions. Hermes. Journal of Linguistics, 31, pp. 171-196.

Burger, M. y Brunner, H. (2000). Scholastic Science Dictionary. USA: Scholastic Inc.

Cabré, M.T. (1999). La terminología. Representación y comunicación. Una teoría de base comunicativa y otros artículos. Barcelona: Institut Universitari de Lingüística Aplicada.

Cabré, M.T. (2010). Terminología y buenas prácticas, Atti Convegno Assiterm 2009, Publifarum, 12. Disponible en línea: http://publifarum.farum.it/ezine_articles.php?id=161. http://dx.doi.org/10.1075/tlrp.1

Carleton, L. y Marzano, R. (2010). Vocabulary games for the classroom. Bloomington: Marzano Reseach Laboratory.

Carretero, M. (1993). Constructivismo y educación. Buenos Aires: Aique.

Carvalho, O. (2011). Dicionários escolares: definiçao oracional e texto lexicográfico. En O. Carvalho y M. Bagno (orgs.) (2011). Dicionários escolares: politicas, formas e usos. Sao Paulo: Parábola.

Carvalho, O. y Bagno, M. (orgs.) (2011). Dicionários escolares: politicas, formas e usos. Sao Paulo: Parábola.

Castillo, A. y García Platero, J.M. (2003). La lexicografía didáctica. En A. Media. La lexicografía española. Barcelona: Ariel, pp. 333-351.

CowIE, A.P. (2000). English Dictionaries for Foreign Learners: A History. Oxford: Oxford University Press.

Cruïlla (2004). Diccionari bàsic de la llengua catalana. Barcelona: Cruïlla.

De Posada, J.M. (1996). Hacía una teoría de las ideas científicas de los alumnos: influencia del contexto. Enseñanza de ciencias, 14 (3), pp. 303-314.

De Vecchi, G. y Giordan, A. (1996). L'Enseignement scientifique. Comment faire pour que «ça marche»? Nice: Z'éditions.

Driver, R. (1983). The pupil as scientist. Milton Keynes: Open University Press.

Eather, J. (2013). A Maths Dictionary for kids. Disponible en línea: http://www.amathsdictionaryforkids.com/.

Enciclopèdia Catalana (1995). DIDAC. Diccionari de català. Barcelona: Enciclopèdia catalana.

EstopÀ, R. (2011). Jugant a definir la ciència: un diccionari de mots de ciència fet per i per a nens i nenes. Terminàlia, 4, pp. 25-33.

Estopà, R. (dir.) (2012). Petit diccionari de ciència. Barcelona: Publicacions de l'Abadia de Montserrat. Estopì, R. (dir.) (2013). Mi primer diccionario de ciencia. Barcelona: Publicacions de l'Abadia de Montserrat. 
EstopÀ, R. (2013). Juguem a definir la ciència: recursos per treballar el lèxic especialitzat a l'escola. En M.A. Sànchez Fèrriz (2013). La terminologia en les ciències de la vida, en la química i en el món educatiu. Barcelona: SCATERM, Memòries de la Societat catalana de terminologia, 4, pp. 199-209.

Estopà, R. y Cornudella, M. (2013). El CLUB LEXIC y el MICROSCOPIO, plataformas en línea para construir diccionarios científicos colaborativos en un proyecto universidad-escuela. Girona: Univest 2013.

Estopà, R. y VAlero, T. (2002). Adquisición del conocimiento especializado y unidades de significación especializada en medicina. En Panace@. Boletín de Medicina y Traducción, 3 (9-10), pp. 277 292. Disponible en línea: http://www.medtrad.org/panacea.html.

Eumo (1990). Primer diccionari. Vic: Eumo editorial.

Fitzgerals, T. (2011). Math Dictionary for kids. USA: Prufock Press.

Glover, D. (2011). Junior Science Dictionary (Maths and Science Dictionaries). Londres: Pearson Education.

Golombek, D. (2006). Prólogo. En G. Charpak et al. Los niños y la ciencia. La aventura de La mano en la masa. Buenos Aires: Siglo XXI editores.

Gutiérrez, B. (1998). La ciencia empieza en la palabra. Barcelona: Península.

Hernández, H. (1989). Los diccionarios de orientación escolar. Contribución al estudio de la lexicografía española. Tubingen: Max Niemeyer.

Ilari, R. (2008). Introduçâo ao estudo do léxico. Brincando con as palavras. Campinas: Contexto.

IzQuierdo, M. y Aliberas, J. (2004). Pensar, actuar i parlar a la classe de ciències. Per un ensenyament de les ciències racional i raonable. Bellaterra: Universitat Autònoma de Barcelona.

JaCKsOn, H. (2002). Lexicography: an introduction. London: Routledge. http://dx.doi.org/10.4324/9780203467282

Khan, S. (2013). First Illustrated Science Dictionary. Oxford: Usborne.

Krieger, M.G. (2012). Diccionário em sala de aula: guia de estudos e exercicios. Rio de Janeiro: Lexikon.

Landau, S. (1984). Dictionaries: The Art and Craft of Lexicography. Cambridge: Cambridge University Press.

Lemke, J. (1997). Aprender a hablar ciencia. Lenguaje, aprendizaje y valores. Barcelona: Paidós.

Locke, D. (I992). SCIENCE AS WRITING. New Haven: Yale University Press. (Versión en español: La ciencia como escritura. Madrid: Cátedra, 1997).

Maldonado, C. (1998). El uso del diccionario en el aula. Madrid: Arco Libros.

Mallart, J. (2002). Competencias básicas y enseñanzas mínimas del currículum de Lengua. Revista de Educación, 329, pp. 219-298.

Marín, N. (2003). La enseñanza de las ciencias en primaria. Almería: Grupo Editorial Universitario.

Martínez Marín (1991). Los diccionarios escolares del español. Lo que son y lo que deberían ser. En I. Ahumada (ed.) (1991). Diccionarios españoles: contenidos y aplicaciones. Jaén: Universidad de Jaén, pp. 53-70.

Marzano, R. y Pickering, D. (2005). Building academic vocabulary. Teacher's manual. Virginia: Association for Supervisition and Curriculum Development.

Moon, R. (2007). Sinclair, lexicography, and the Cobuild Project the application of theory. International Journal of Corpus Linguistics, 12, pp. 159-181.

http://dx.doi.org/10.1075/ijcl.12.2.05moo

ONDA (2004). Diccionari junior nou. Barcelona: Onda.

Oxford University Press (2001). Oxford First Illustrated Science Dictionary.

Oxford University Press (2001). Oxford First Illustrated Maths Dictionary.

Oxford University Press (2001). Oxford First Science Dictionary.

Oxford University Press (2001). Oxford First Maths Dictionary. 
Oxford University Press (2003). Oxford Primary Illustrated Science Dictionary.

Oxford University Press (2003). Oxford Primary Illustrated Maths Dictionary.

Oxford University Press (2006). Student's Science Dictionary.

Pérez Lagos, F. (1998). Los diccionarios escolares de los últimos años ¿̨una nueva lexicografía didáctica? En M. Alvar y G. Corpas (coords.) (1998). Diccionarios, frases, palabras. Málaga: Universidad de Málaga.

Pierce, R. (2010). Illustrated Mathematics Dictionary Disponible en línea: http://www.mathsisfun. com/aboutmathsisfun.html.

Prado, J. (2000). El diccionario y la enseñanza de la lengua. En S. Ruschtaller y J. Prado (eds.). Tendencias en la investigación lexicográfica del español: el diccioanrio como objeto lingüistico y didáctico. Huelva: Universidad de Huelva, pp. 179-191.

Rey-Debove, J. (1971). Étude linguistique et sémiotique des dictionnaires français contemporains. ParísLa Haya: Mouton. http://dx.doi.org/10.1515/9783111323459

Santillana (2012). Nou Diccionari escolar de la llengua catalana. Barcelona: Santillana Grup Promotor.

Stocker, K. (1964). Principios de didáctica moderna. Buenos Aires: Kapelusz.

TARP, S. (1994). Funktionen in Fachwörterbüchern. Fachlexikographie. Fachwissen und seine Repräsentation. En H. Bergenholtz et al. Wörterbüchern. Tübingen: Gunter Narr Verlag, pp. 229-246.

VOX (2009). Diccionari escolar de la llengua catalana. Barcelona: Larouse Editorial.

Vygotsky, L. (1962). Pensamiento y lenguaje. New York/Cambridge: Wiley and MTT Press.

WATT, M. et al. (1989). Talkink and writing for learning. En D. Bentley y M. Watts (1989). Learning and teaching in school science. Philadelphia: Open University Press.

Westphal, L. (2009). Science Dictionary for kids. Grades 4-9. Texas: Prufrock Press.

WILD, K. et al. (2011). Oxford Children's Corpus: A corpus of writing for children. Poster at ICLIC (International Corpus Linguistics Conference), Birmingham, UK.

Wild, K. et al. (2013). The Oxford Children's Corpus: Using a Children's Corpus in Lexicography. International Journal of Lexicography, 26, 1, pp. 190-218.

http://dx.doi.org/10.1093/ijl/ecs017 


\title{
Build to deconstruct and re-construct: collaborati- ve development of a school science dictionary
}

\author{
Rosa Estopà \\ Institut Universitari de Lingüística Aplicada, Universitat Pompeu Fabra \\ rosa.estopa@upf.edu
}

This paper presents the collaborative methodology used in the creation of a school dictionary of science for children aged between 5 and 8, based on a corpus of definitions given by children in Catalan language.

Children of these ages, also the youngest, often ask (and ask themselves) the meaning of specialised words: what is a crater? What is the telescope used for? And a microscope? Which colour is our brain? Are the universe and space the same? What does neurone mean?... In the first steps of school education, working on academic vocabulary should be a fact.

However, many languages - such as Spanish, Catalan, Galician and Basque- do not have dictionaries available for all school levels. In terms of Lexicography, in Catalan schools there is a lack of dictionaries for children who are starting on literacy. To try to address this gap, our aim was to construct a dictionary on the more general scientific terms that are starting to be used in the first primary school year, that is to say, for boys and girls aged between 5 and 8 .

The work was conducted over a period of 3 years with 500 children from 3 schools, who defined and described the most basic terms of science, such as sun, air, water, star, time, brain, crater, telescope, etc. First, we created a corpus of children explanations and drawings: el IULATERM Children's Corpus (ICC), because the maximum suitability of a dictionary to its final audience can be based on the cumulative knowledge of a group representative of the body of schoolchildren who will use it.

Then, from the corpus, we selected only the accumulated information which was scientifically positive and thus produced definitions and explanations, without adding new information. We selected as well a drawing for each definition made by the students.

The Petit diccionari de ciència (2012) -also published in Spanish by Publicacions de l'Abadia de Montserrat (2013), is a specialised type I dictionary that contains 100 basic terms corresponding to 126 different meanings. Each entry includes the grammatical category, an explanation and a drawing. In this dictionary, the explanation of the term is an account of how the child defines this word, of how he or she understands it, of what it means to him or her. It is barely a canonical definition; it is always sentence-based and composed of more than one sentence. The information is ordered according to the importance children have given to semantic characteristics in their definitions. It often follows patterns such as ' $\mathrm{X}$ is a' (normally followed by a hypernym and not always at the same level) or 'When..,' (usually to explain the meaning of a verb).Here are some examples of how the definitions begin:

air

Air is transparent and colourless ...

compass

A compass is an object similar to a clock ...

Finally, the article demonstrates the suitability of the dictionary through the results of a lexical comprehension test carried out with 160 students. We compare 4 scholar dictionaries. Our dictionary, The Petit diccionari de ciència (My First Dictionary of Science), with the tree following:

ENCICLOPÈDIA CATALANA (1995) DIDAC. Diccionari de català.

EUMO Editorial (1990). Primer diccionari.

SANTILLANA Grup Promotor. (2012) Nou Diccionari escolar de la llengua catalana.

We ask them to select which definition they understand best and which definition they understand worst. We also ask them to mark all the words of the definitions that they can understand. The experiment shows that our dictionary was their favourite and it was also the dictionary with less marked words by the children.

The accumulative and collaborative methodology, based on a corpus of children's definitions to construct the dictionary, is shown adequate: first build, then deconstruct, and finally re-construct. The children build their definition individually, we deconstruct all of them and we accumulate the knowledge, and in the end we select the information which is scientifically relevant. PDC's definitions are explanations based on the controlled accumulative knowledge of children aged 5 to 8 . Moreover, the experimental test has ratified that 5 to 8 -year-old children understand better the PDC when compared to other Catalan school dictionaries with -from our point of view- a high school level. These results allow us to develop our future plans, which work the language together with scientific knowledge from the beginning: there is no science without the words to represent it or convey it. 